\title{
EFFECTS OF HALL CURRENT ON UNSTEADY HYDROMAGNETIC FREE CONVECTION FLOW PAST AN IMPULSIVELY MOVING VERTICAL PLATE WITH NEWTONIAN HEATING
}

\author{
G.S. SETH* ${ }^{*}$ S. SARKAR and R. SHARMA \\ Department of Applied Mathematics \\ Indian School of Mines \\ Dhanbad 826004, INDIA \\ E-mail: gsseth_ism@yahoo.com
}

\begin{abstract}
An investigation of unsteady hydromagnetic free convection flow of a viscous, incompressible and electrically conducting fluid past an impulsively moving vertical plate with Newtonian surface heating embedded in a porous medium taking into account the effects of Hall current is carried out. The governing partial differential equations are first subjected to the Laplace transformation and then inverted numerically using INVLAP routine of Matlab. The governing partial differential equations are also solved numerically by the Crank-Nicolson implicit finite difference scheme and a comparison has been provided between the two solutions. The numerical solutions for velocity and temperature are plotted graphically whereas the numerical results of skin friction and the Nusselt number are presented in tabular form for various parameters of interest. The present solution in special case is compared with a previously obtained solution and is found to be in excellent agreement.
\end{abstract}

Key words: hydromagnetic free convection flow, Newtonian heating, Hall current, porous medium, INVLAP routine in Matlab.

\section{Introduction}

Theoretical/ experimental investigation of hydrodynamic free convection flow from a solid body with different geometries embedded in a porous medium has received considerable attention during the past decades due to its varied and wide applications in several areas of science and technology such as geothermal reservoirs, thermal insulators, chemical catalytic reactors, grain storage, food processing, energy efficient drying of porous solids, heat exchanger devices, nuclear waste repositories, enhanced recovery of oil and gas, underground energy transport, etc. The most basic problem of natural convection in porous media is the boundary layer flow along a heated vertical flat plate embedded in a fluid-saturated porous medium which was investigated by Cheng and Minkowycz [1]. They obtained similarity solution for the case when the wall temperature varies as a power function of the distance from the leading edge. Nakayama and Koyama [2] analyzed combined free and forced convection flow in Darcian and non-Darcian porous media. Lai and Kulacki [3] studied non-Darcy mixed convection flow along a vertical wall in a fluid saturated porous medium. Bakier et al. [4] obtained non-similar solution for free convection flow along a vertical porous plate embedded in a fluid saturated porous medium in the presence of internal heat generation using the finite difference technique. Comprehensive reviews of thermal convection in porous media are well presented in the form of books and monograms by Nield and Bejan [5], Ingham and Pop [6], Vafai [7], Pop and Ingham [8], Ingham et al. [9] and Bejan et al. [10].

It is well known that heat transfer characteristics are dependent on the thermal boundary conditions. Newtonian heating is a kind of wall-to-ambient heating process where the rate of heat transfer from the

\footnotetext{
* To whom correspondence should be addressed
} 
bounding surface with a finite heat capacity is proportional to the local surface temperature and it is usually called conjugate convective flow. This type of situation occurs in important engineering devices such as in heat exchangers, gas turbines and also in convective flows set up when the bounding surfaces absorb heat by solar radiation (seasonal thermal energy storage systems). Therefore, the interaction of conductionconvection coupled effects is of much significance from a practical point of view and it must be considered when evaluating the conjugate heat transfer processes in many engineering applications. Merkin [11] initiated the study of free convection boundary layer flow over a vertical surface with Newtonian heating while Lesnic et al. [12], [13] considered free convection boundary layer flow along vertical and horizontal surfaces in a porous medium generated by Newtonian heating. Lesnic et al. [14] also investigated free convection boundary layer flow over a nearly horizontal surface in a porous medium with Newtonian heating. Salleh et al. [15] discussed forced convection boundary layer flow at a forward stagnation point with Newtonian heating. Subsequently, Salleh et al. [16] considered steady boundary layer flow over a stretching sheet with Newtonian heating. Chaudhary and Jain [17] investigated unsteady free convection flow past an impulsively started vertical plate with Newtonian heating. Mebine and Adigio [18] studied unsteady free convection flow of a viscous, incompressible and optically thin radiating fluid past an impulsively started vertical porous plate with Newtonian heating. Narahari and Ishak [19] investigated the influence of thermal radiation on unsteady free convection flow of an optically thick fluid past a moving vertical plate with Newtonian heating. They considered three cases of interest, namely, (i) impulsive movement of the plate; (ii) uniformly accelerated movement of the plate and (iii) exponentially accelerated movement of the plate. Narahari and Nayan [20] analyzed unsteady free convection flow of an optically thick fluid past an impulsively moving infinite vertical plate with Newtonian heating in the presence of thermal radiation and mass diffusion. Olanrewaju and Makinde [21] investigated boundary layer stagnation point flow of a nanofluid over a permeable flat surface with Newtonian heating.

However, in all these investigations, the effects of the magnetic field are not taken into account. Investigation of unsteady hydromagnetic convective boundary layer flow of electrically conducting fluids in porous and non-porous media is of much significance due to its varied and wide applications in many areas of science and engineering, viz. boundary layer flow control, plasma studies, geothermal energy extraction, in the field of solar energy collection, cooling of an infinite metallic plate in a cooling bath, magnetohydrodynamic (MHD) stirring of molten metal, magnetic levitation and casting, MHD marine propulsion and on the performance of many engineering devices, namely, MHD power generators (Steg and Sutton [22]; Womac [23]), MHD flow-meters (Shercliff [24]), MHD pumps (Blake [25]), MHD accelerators (Marston [26]), controlled thermonuclear reactors (Christofilos [27]), etc. Raptis [28] investigated unsteady two-dimensional natural convection flow of an electrically conducting, viscous and incompressible fluid along an infinite vertical plate embedded in a porous medium. Jha [29] studied hydromagnetic free convection and mass transfer flow past a uniformly accelerated moving vertical plate through a porous medium when magnetic field is fixed with the moving plate. Chamkha [30] analyzed unsteady MHD free convection flow through a porous medium supported by a surface. Kim [31] investigated unsteady MHD free convection flow past a moving semi-infinite vertical porous plate embedded in a porous medium with variable suction. Ibrahim et al. [32] analyzed unsteady hydromagnetic free convection flow of micro-polar fluid and heat transfer past a vertical porous plate through a porous medium in the presence of thermal and mass diffusions with a constant heat source. Chamkha [33] considered unsteady MHD free convection flow with heat and mass transfer past a semi-infinite vertical permeable moving plate in a uniform porous medium with heat absorption. Seth et al. [34] obtained an exact solution of unsteady MHD natural convection flow with radiative heat transfer past an impulsively moving vertical plate with ramped temperature embedded in a porous medium. Mahmoud [35] discussed the effects of thermal radiation on unsteady MHD free convection flow past an infinite vertical porous plate taking into account the effects of viscous dissipation. Ogulu and Makinde [36] considered unsteady hydromagnetic free convection flow of a dissipative and radiative fluid past a vertical plate with constant heat flux. Chamkha et al. [37] investigated unsteady MHD natural convection flow from a heated vertical porous plate in a micropolar optically thin fluid with Joule heating, chemical reaction and radiation effects. They obtained solution of the governing equations by the implicit finite difference scheme. Mohamed et al. [38] discussed unsteady MHD free convection heat and 
mass transfer flow of an optically thick radiating fluid past an impulsively moving infinite hot vertical plate in a porous medium in the presence of chemical reaction and heat sink. Singh and Makinde [39] investigated steady hydromagnetic free convection flow along an inclined plate with Newtonian heating in the presence of volumetric heat generation. Recently, Abid et al. [40] studied unsteady MHD free convection flow in a porous medium with constant mass diffusion and Newtonian heating.

However, in all the above investigations, effects of Hall current are not taken into account. If an ionized fluid with low density is subjected to a strong magnetic field then the electrical conductivity normal to the magnetic field is lowered owing to free spiraling of electrons and ions about the magnetic lines of force prior to collision and a current is thereby generated which is mutually perpendicular to electric and magnetic fields. This current is known as Hall current. It plays an important role in determining flow features of the fluid flow problems because it induces secondary flow in the flow-field (Sherman and Sutton [41]). The study of MHD viscous flows with Hall current has significant applications in several areas of engineering problems, viz. the development of efficient Hall thrusters in magnetic propulsion (Fife [42]), plasma actuator control of hypersonic flows (Shang et al. [43]), influence of Hall effects on the characteristics of MHD generators (Kholshchevnikova [44]) and superconducting films (Michaeli [45]) etc. The current trend is towards the application of a strong magnetic field (i.e., in materials processing (Davidson [46]), MHD energy system (Hardianto et al. [47]), MHD sea water propulsion (Mathon [48]) etc.) and towards a low density of the gas (i.e., in application of plasma devices for future vehicle systems (Van Wie [49]), nuclear fission research (Morley et al. [50] etc.). Significant contributions to hydromagnetic free convection flow past a flat plate with Hall effects under different thermal conditions were made by several researchers in the past. Mention may be made of the research studies of Pop and Watanabe [51], AboEldahab and Elbarbary [52], Takhar et al. [53] and Saha et al. [54]. Recently, Seth et al. [55] studied the effects of Hall current and rotation on unsteady MHD natural convection flow of a viscous, incompressible, electrically conducting and heat absorbing fluid past an impulsively moving vertical plate with ramped temperature in a porous medium in the presence of thermal diffusion.

The objective of the present investigation is to study unsteady hydromagnetic free convection flow of an electrically conducting, viscous and incompressible fluid past a vertical plate embedded in a porous medium taking into account the effects of Hall current when the fluid flow is generated due to impulsive movement of the vertical plate with Newtonian heating. According to the best of authors' knowledge this problem has not yet received attention of researchers though it is significantly important in science and engineering. A comparison of the present solution for fluid velocity in the absence of magnetic field, Hall current and permeability of medium is made with the exact solution obtained by Chaudhary and Jain [17]. It was found that our result is in excellent agreement with that of Chaudhary and Jain [17].

\section{Formulation of the problem and its solution}

Consider an unsteady free convection flow of a viscous, incompressible and electrically conducting fluid past an infinite vertical plate embedded in a uniform porous medium. The $x^{\prime}$-axis is taken along the plate in the upward direction and the $y^{\prime}$-axis normal to the plane of plate in the fluid. The fluid is permeated by a uniform transverse magnetic field $B_{0}$ which is applied in a direction parallel to the $y^{\prime}$-axis. Initially, i.e., at time $t^{\prime} \leq 0$, both the fluid and plate are at rest and at a uniform temperature $T_{\infty}^{\prime}$. At time $t^{\prime}>0$, the plate starts moving in the $x^{\prime}$ direction with uniform velocity $U_{0}$ against gravitational field in its own plane. At the same time, heat transfers from the plate to the fluid, which is directly proportional to the local surface temperature. Since the plate is of infinite extent along $x^{\prime}$ and $z^{\prime}$ directions and is electrically non-conducting, all physical quantities depend on $y^{\prime}$ and $t^{\prime}$ only. The geometry of the problem is shown in Fig.1. The induced magnetic field generated by the fluid motion is neglected in comparison to the applied one. This assumption is justified because the magnetic Reynolds number is very small for liquid metals and partially ionized fluids (Cramer and Pai [56]). Also no external electric field is applied so the effect of 
polarization of the fluid is negligible. This corresponds to the case where no energy is added or extracted from the fluid by electrical means (Cramer and Pai [56]).

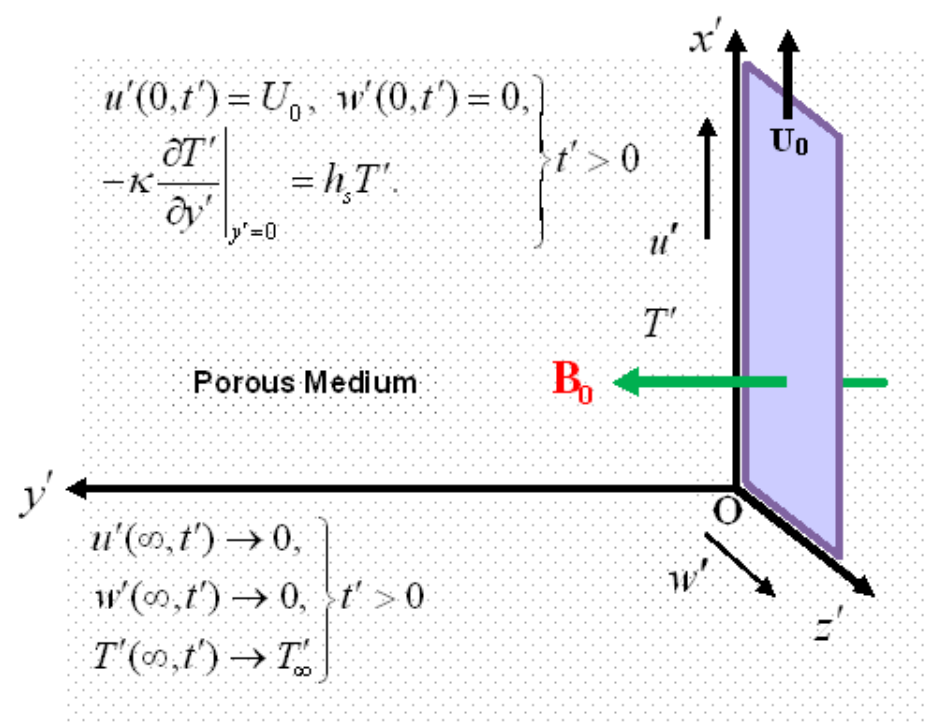

Fig.1. Geometry of the problem.

Taking into consideration the assumptions made above, the governing equations for free convection flow of a viscous, incompressible and electrically conducting fluid through a uniform porous medium taking Hall current into account, under Boussinesq approximation, are given by

$$
\begin{aligned}
& \frac{\partial u^{\prime}}{\partial t^{\prime}}=v \frac{\partial^{2} u^{\prime}}{\partial y^{\prime 2}}-\frac{\sigma B_{0}^{2}}{\rho\left(1+m^{2}\right)}\left(u^{\prime}+m w^{\prime}\right)-\frac{v u^{\prime}}{K_{l}^{\prime}}+g \beta^{\prime}\left(T^{\prime}-T_{\infty}^{\prime}\right), \\
& \frac{\partial w^{\prime}}{\partial t^{\prime}}=v \frac{\partial^{2} w^{\prime}}{\partial y^{\prime 2}}+\frac{\sigma B_{0}^{2}}{\rho\left(1+m^{2}\right)}\left(m u^{\prime}-w^{\prime}\right)-\frac{v w^{\prime}}{K_{l}^{\prime}}, \\
& \rho c_{p} \frac{\partial T^{\prime}}{\partial t^{\prime}}=\kappa \frac{\partial^{2} T^{\prime}}{\partial y^{\prime 2}}
\end{aligned}
$$

where $u^{\prime}, w^{\prime}, v, \rho, \sigma, m=\omega_{e} \tau_{e}, \omega_{e}, \tau_{e}, g, \beta^{\prime}, T^{\prime}, c_{p}, \kappa$ and $K_{l}^{\prime}$ are, respectively, fluid velocity in $x^{\prime}-$ direction, fluid velocity in $z^{\prime}$-direction, kinematic coefficient of viscosity, fluid density, electrical conductivity, Hall current parameter, cyclotron frequency, electron collision time, acceleration due to gravity, volumetric coefficient of thermal expansion, fluid temperature, specific heat at constant pressure, thermal conductivity of fluid and permeability of porous medium.

The initial and boundary conditions for the problem are 


$$
\left.\begin{array}{llll}
t^{\prime} \leq 0 & : u^{\prime}=0, w^{\prime}=0, \quad T^{\prime}=T_{\infty}^{\prime} & \text { for } & y^{\prime} \geq 0, \\
t^{\prime}>0 & : u^{\prime}=U_{0}, w^{\prime}=0, \frac{\partial T^{\prime}}{\partial y^{\prime}}=-\frac{h_{s}}{\kappa} T^{\prime} & \text { at } & y^{\prime}=0, \\
& u^{\prime} \rightarrow 0, \quad w^{\prime} \rightarrow 0, T^{\prime} \rightarrow T_{\infty}^{\prime} & \text { as } & y^{\prime} \rightarrow \infty .
\end{array}\right\}
$$

where $h_{s}$ is the heat transfer coefficient.

Equations (2.1) to (2.3), in a non-dimensional form, assume the following form

$$
\begin{aligned}
& \frac{\partial u}{\partial t}=\frac{\partial^{2} u}{\partial y^{2}}-\frac{M^{2}}{\left(1+m^{2}\right)}(u+m w)-\frac{u}{K_{l}}+\mathrm{G}_{r} T, \\
& \frac{\partial w}{\partial t}=\frac{\partial^{2} w}{\partial y^{2}}+\frac{M^{2}}{\left(1+m^{2}\right)}(m u-w)-\frac{w}{K_{l}}, \\
& \frac{\partial T}{\partial t}=\frac{1}{\mathrm{P}_{r}} \frac{\partial^{2} T}{\partial y^{2}}
\end{aligned}
$$

where

$$
\begin{aligned}
& y=y^{\prime} U_{0} / v, \quad u=u^{\prime} / U_{0}, \quad w=w^{\prime} / U_{0}, \quad t=t^{\prime} U_{0}^{2} / \mathrm{v}, \\
& M^{2}=\sigma B_{0}{ }^{2} v / \rho U_{0}^{2}, \quad K_{1}=K_{l}{ }^{\prime} U_{0}{ }^{2} / v^{2}, \\
& T=\left(T^{\prime}-T_{\infty}^{\prime}\right) / T_{\infty}^{\prime}, \quad \mathrm{G}_{r}=g \beta^{\prime} v T_{\infty}^{\prime} / U_{0}^{3} \quad \text { and } \quad \mathrm{P}_{r}=v \rho c_{p} / \kappa
\end{aligned}
$$

$u, w, T, M^{2}, K_{l}, \mathrm{G}_{r}$ and $\mathrm{P}_{r}$ are, respectively, the dimensionless primary fluid velocity, dimensionless secondary fluid velocity, dimensionless fluid temperature, magnetic parameter, permeability parameter, thermal Grashof number and Prandtl number.

The initial and boundary conditions (2.4), in a non-dimensional form, become

$$
\begin{array}{cl}
t \leq 0 \quad: u=0, \quad w=0, \quad T=0, \quad \text { for } \quad y \geq 0, \\
t>0 \quad: u=1, \quad w=0, \quad \frac{\partial T}{\partial y}=-\gamma(1+T), \quad \text { at } \quad y=0, \\
u \rightarrow 0, \quad w \rightarrow 0, \quad T \rightarrow 0, \quad \text { as } \quad y \rightarrow \infty,
\end{array}
$$

where $\gamma=h_{s} v / U_{0}$ is the Newtonian heating parameter (Abid et al. [57], [40]). It is worth mentioning that $\gamma=0$ corresponds to having $h_{s}=0$, i.e., an insulated wall is present and when $\gamma \rightarrow \infty$, the wall temperature remains constant (Salleh et al. [16]; Abid et al. [57]).

Equations (2.5) and (2.6) are presented, in a compact form, as 


$$
\frac{\partial F}{\partial t}=\frac{\partial^{2} F}{\partial y^{2}}-\lambda F+\mathrm{G}_{r} T
$$

where

$$
F=u+i w \quad \text { and } \quad \lambda=M^{2}(1-i m) /\left(1+m^{2}\right)+1 / K_{l} .
$$

Initial and boundary conditions (2.8a) to (2.8c), in a compact form, become

$$
\begin{aligned}
& F=0, \quad T=0 \quad \text { for } \quad y \geq 0 \quad \text { and } \quad t \leq 0, \\
& F=1, \quad \frac{\partial T}{\partial y}=-\gamma(1+T) \quad \text { at } \quad y=0 \text { for } \quad t>0, \\
& F \rightarrow 0, \quad T \rightarrow 0 \quad \text { as } \quad y \rightarrow \infty \quad \text { for } \quad t>0 .
\end{aligned}
$$

Equations (2.7) and (2.9), after taking the Laplace transform and using initial conditions (2.10a), reduce to

$$
\begin{aligned}
& \frac{d^{2} \bar{T}}{d y^{2}}-\operatorname{Pr} s \bar{T}=0, \\
& \frac{d^{2} \bar{F}}{d y^{2}}-(s+\lambda) \bar{F}+\mathrm{G}_{r} \bar{T}=0,
\end{aligned}
$$

where $\bar{T}(y, s)=\int_{0}^{\infty} T(y, t) e^{-s t} d t, \quad \bar{F}(y, s)=\int_{0}^{\infty} F(y, t) e^{-s t} d t$ and $s>0 \quad(s$ being the Laplace transform parameter).

Boundary conditions (2.10b) and (2.10c), after taking the Laplace transform, become

$$
\begin{aligned}
& \bar{F}=1 / s, \quad \frac{d \bar{T}}{d y}=-\gamma\left(\frac{1}{s}+\bar{T}\right) \text { at } \quad y=0, \\
& \bar{F} \rightarrow 0, \quad \bar{T} \rightarrow 0, \quad \text { as } \quad y \rightarrow \infty .
\end{aligned}
$$

Solutions of Eqs (2.11) and (2.12) subject to the boundary conditions (2.13a) and (2.13b) are given by

$$
\begin{aligned}
& \bar{T}(y, s)=\frac{\gamma e^{-y \sqrt{\operatorname{Pr} s}}}{s(\sqrt{\operatorname{Pr} s}-\gamma)}, \\
& \bar{F}(y, s)=\frac{1}{s} e^{-y \sqrt{s+\lambda}}-\frac{G_{I} \gamma}{s\left(s+\lambda_{3}\right)\left(\sqrt{\mathrm{P}_{r} s}-\gamma\right)}\left\{e^{-y \sqrt{s+\lambda}}-e^{-y \sqrt{\mathrm{P}_{r} s}}\right\},
\end{aligned}
$$

where

$$
G_{l}=\mathrm{Gr} /\left(1-\mathrm{P}_{r}\right) \quad \text { and } \quad \lambda_{3}=\lambda /\left(1-\mathrm{P}_{r}\right)
$$


The exact solution of the fluid temperature $T(y, t)$ can be obtained by taking the inverse Laplace transform of Eq. (2.14) and is provided by Chaudhary and Jain [17] when $\gamma=1$. Also, Chaudhary and Jain [17] obtained the inverse Laplace transform of Eq.(2.15) when $\lambda=0$ and $\gamma=1$. We have obtained the inverse Laplace transform of Eq. (2.15) for the case $\lambda \neq 0$ using INVLAP routine in Matlab (de Hogg et al. [58]; Hollenbeck [59]). A numerical solution of fluid velocity obtained using INVLAP routine for the case when the magnetic field, porous medium and Hall current is absent, is compared with the exact solution presented by Chaudhary and Jain [17].

\section{Numerical solution}

Equations (2.5) and (2.6) subject to the initial and boundary conditions (2.8) can also be solved numerically using the Crank-Nicolson implicit finite difference scheme. Therefore, we have obtained a numerical solution of this problem.

The finite difference equations corresponding to the governing Eqs (2.5) to (2.7) are as follows

$$
\begin{aligned}
& \frac{u_{i, j+1}-u_{i, j}}{\Delta t}=\frac{u_{i-1, j+1}-2 u_{i, j+1}+u_{i+1, j+1}+u_{i-l, j}-2 u_{i, j}+u_{i+1, j}}{2(\Delta y)^{2}}+ \\
& -\frac{M^{2}}{1+m^{2}}\left(u_{i, j}+m w_{i, j}\right)-\frac{u_{i, j}}{K_{1}}+\mathrm{G}_{r} T_{i, j}, \\
& \frac{w_{i, j+1}-w_{i, j}}{\Delta t}=\frac{w_{i-1, j+1}-2 w_{i, j+1}+w_{i+1, j+1}+w_{i-1, j}-2 w_{i, j}+w_{i+1, j}}{2(\Delta y)^{2}}+ \\
& +\frac{M^{2}}{1+m^{2}}\left(m u_{i, j}-w_{i, j}\right)-\frac{w_{i, j}}{K_{1}}, \\
& \frac{T_{i, j+1}-T_{i, j}}{\Delta t}=\frac{1}{\mathrm{P}_{r}} \frac{T_{i-1, j+1}-2 T_{i, j+1}+T_{i+1, j+1}+T_{i-1, j}-2 T_{i, j}+T_{i+1, j}}{2(\Delta y)^{2}} .
\end{aligned}
$$

Initial and boundary conditions (2.8) become

$$
\begin{aligned}
& u_{i, 0}=0, \quad w_{i, 0}=0, \quad T_{i, 0}=0, \\
& u_{0, j}=1, \quad w_{0, j}=0, \quad \frac{T_{1, j}-T_{0, j}}{\Delta y}=-\gamma\left(1+T_{0, j}\right), \\
& u_{\max , j}=0, \quad w_{\max , j}=0, \quad T_{\max , j}=0,
\end{aligned}
$$

where suffix $i$ corresponds to $y$ and suffix $j$ corresponds to $t$. Also, $\Delta t=t_{j+1}-t_{j}$ and $\Delta y=y_{i+1}-y_{i}$. The region under consideration is restricted to a rectangle of finite dimensions with $y_{\max }=6$ (corresponding to $y \rightarrow \infty)$ and $t_{\max }=2$ where $\Delta y=0.025$ and $\Delta t=0.0025$ such that the computational domain is divided into $241 \times 801$ grid points. The assumptions of $y_{\max }=6$ and $t_{\max }=2$ are justified since boundary condition (3.4c) is satisfied within tolerance limit of $10^{-4}$. The mesh size $\Delta y=0.025$ with time step $\Delta t=0.0025$ is 
finalized after comparing the results in this case with the results obtained when mesh size is reduced to $50 \%$ of the present case and it is noticed that the difference between these two results is less than half a unity in the fourth decimal place. The finite difference equations at each $j^{\text {th }}$ level constitute a tridiagonal system of equations which are solved by Thomas algorithm as given in Carnahan et al. [60]. A numerical solution for the fluid temperature and fluid velocity is obtained corresponding to the desired degree of accuracy for required time by performing computations for a number of time steps. It was found that the absolute difference between the numerical values of fluid temperature and fluid velocity obtained for two consecutive time steps is less than $10^{-4}$ Hence the scheme designed is stable. Moreover, the CrankNicolson method has a local truncation error of $O\left\{(\Delta y)^{2}+(\Delta t)^{2}\right\}$ which tends to zero as $\Delta y$ and $\Delta t$ tends to zero which justifies consistency (Antia [61], pp.643-644). Stability and consistency together ensure convergence of the scheme.

The primary skin friction $\tau_{x}$ and secondary skin friction $\tau_{z}$ are given by

$$
\tau_{x}=\left.\frac{\partial u}{\partial y}\right|_{y=0} \quad \text { and } \quad \tau_{z}=\left.\frac{\partial w}{\partial y}\right|_{y=0}
$$

The Nusselt number $\mathrm{N}_{\mathrm{u}}$ is given by

$$
\mathrm{N}_{\mathrm{u}}=\left.\frac{\partial T}{\partial y}\right|_{y=0}
$$

The numerical values of skin friction and Nusselt number are obtained numerically using computed values of fluid velocity and fluid temperature, respectively. It may be noted that the derivatives involved in Eqs (3.5) and (3.6) are evaluated using five point forward difference formula for the first order derivative (Antia [61], page 161).

\subsection{Validation of numerical solution}

In order to validate our numerical scheme we have presented a comparison of the results obtained numerically with the exact results. The expression for the Nusselt number $\mathrm{Nu}$ is obtained using the exact solution for the fluid temperature (Chaudhary and Jain [17]) which is given by

$$
\mathrm{Nu}=e^{t / \operatorname{Pr}} \operatorname{erfc}\left(-\sqrt{\frac{t}{\operatorname{Pr}}}\right) .
$$

We have presented in Tab.1 a comparison between the numerical values of the Nusselt number obtained using the INVLAP routine of Matlab and the finite difference scheme mentioned above with the exact values obtained from expression (3.7). It is evident from Tab.1 that the numerical values of the Nusselt number obtained through the finite difference scheme are in good agreement with the values of the Nusselt number obtained by the INVLAP routine of Matlab. Moreover, it is also noticed from Tab.1 that numerical values for the Nusselt number are in excellent agreement with the exact values of the Nusselt number obtained from Eq.(3.7). 
Table 1. Nusselt number $-\mathrm{Nu}$ when $\gamma=1$.

\begin{tabular}{|c|c|c|c|c|c|c|c|c|c|}
\hline \multirow{2}{*}{$\begin{array}{c}\mathrm{Pr} \rightarrow \\
t \downarrow\end{array}$} & \multicolumn{2}{|c|}{ Result by Finite Difference } & \multicolumn{2}{c|}{ Result by INVLAP routine } & \multicolumn{3}{c|}{ Exact Result } \\
\hline 0.3 & 0.5 & 0.71 & 0.3 & 0.5 & 0.71 & 0.3 & 0.5 & 0.71 \\
\hline 0.5 & 5.0084 & 3.1459 & 2.5052 & 5.009 & 3.1462 & 2.5055 & 5.009 & 3.1462 & 2.5055 \\
\hline 0.7 & 20.261 & 5.0084 & 3.5683 & 10.2295 & 5.0090 & 3.5687 & 10.2295 & 5.0089 & 3.5686 \\
\hline
\end{tabular}

In Fig. 2 we have also presented a comparison between the exact values of fluid velocity obtained in the absence of Hall current, magnetic field and porous medium (Chaudhary and Jain [17]) with the numerical values of fluid velocity obtained by the Crank-Nicolson implicit finite difference scheme and by INVLAP routine of Matlab. It is seen that there is an excellent agreement between these solutions. This justifies the correctness of the results presented in the manuscript.

\section{Results and discussion}

In order to analyze the effects of the thermal buoyancy force, magnetic field, Hall current, permeability of the medium, Newtonian heating, thermal diffusivity and time on the flow-field, a numerical solution of the primary fluid velocity $u(y, t)$ and secondary fluid velocity $w(y, t)$ is depicted graphically versus the boundary layer coordinate $y$ in Figs 2 to 9 for various values of the Grashof number $G_{r}$, magnetic parameter $M^{2}$, Hall current parameter $m$, permeability parameter $K_{l}$, Newtonian heating parameter $\gamma$, Prandtl number $P_{r}$ and time $t$. It is revealed from the Figs 2 to 9 that the primary and secondary fluid velocities attain maximum value near the surface of the plate and then decrease properly on increasing the boundary layer coordinate $y$ to approach the free stream value.

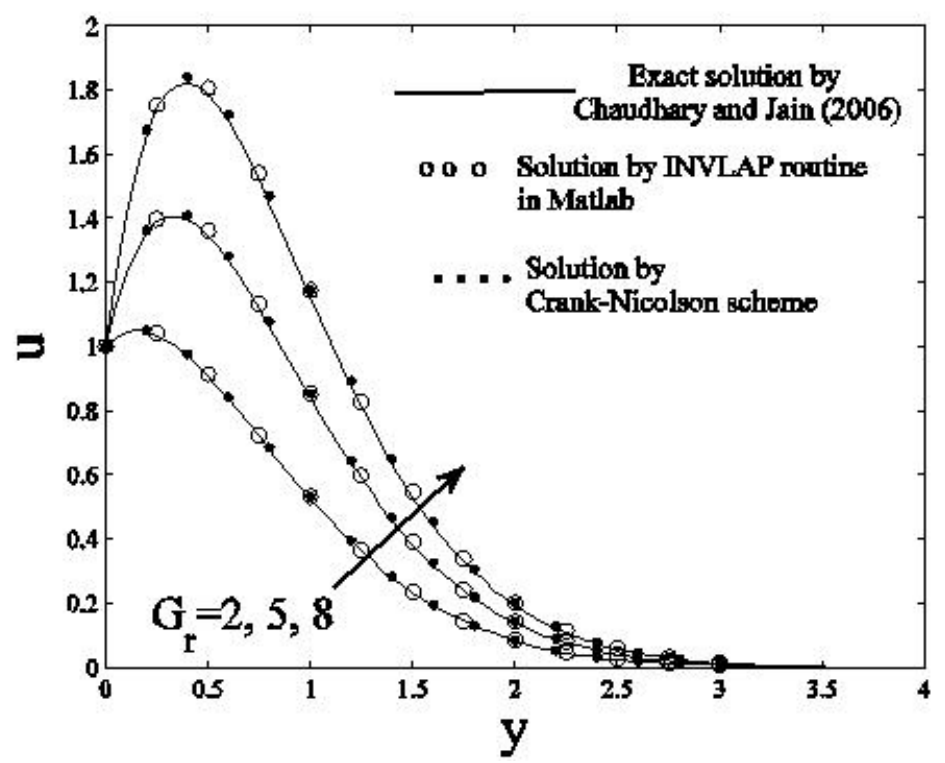

Fig.2. Primary velocity profiles when $M^{2}=0, m=0,1 / K_{1}=0, \gamma=1, \mathrm{P}_{r}=0.71$ and $t=0.5$. 


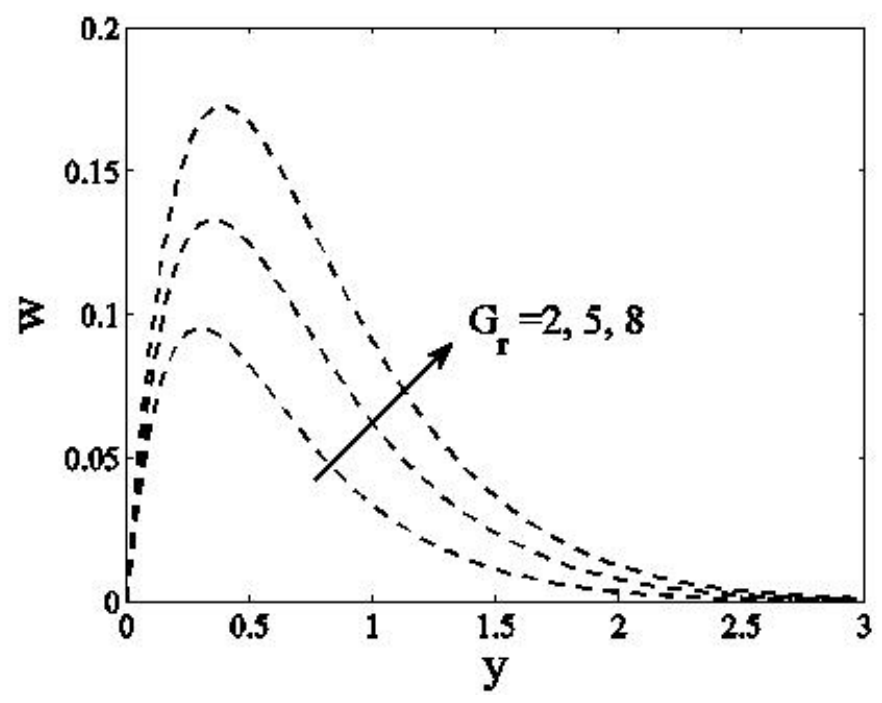

Fig.3. Secondary velocity profiles when $M^{2}=15, m=0.5, K_{l}=0.4, \gamma=1, \mathrm{P}_{r}=0.71$ and $t=0.5$.

Figures 2 and 3 demonstrate the effects of the Grashof number $\mathrm{G}_{\mathrm{r}}$ on the primary fluid velocity $u$ and secondary fluid velocity $w$, respectively. It is noticed from Figs 2 and 3 that both $u$ and $w$ increase on increasing $G_{r}$. $G_{r}$ represents the relative strength of the thermal buoyancy force to the viscous force, $G_{r}$ increases on increasing the strength of the thermal buoyancy force. This implies that the thermal buoyancy force tends to accelerate the fluid flow in both the primary and secondary flow directions throughout the boundary layer region. Figure 4 illustrates the influence of the magnetic field on the primary and secondary fluid velocities. It is revealed from Fig.4 that $u$ decreases on increasing $M^{2}$. With an increase in $M^{2}$, the secondary fluid velocity $w$ increases in a region near the plate and then decreases in the region away from the plate. $M^{2}$ signifies the relative strength of the magnetic force to the viscous force, $M^{2}$ increases on increasing the strength of the magnetic force. This implies that the magnetic field tends to retard the fluid flow in the primary flow direction throughout the boundary layer region whereas it tends to retard the fluid flow in the region away from the plate in the secondary flow direction. However, the magnetic field tends to accelerate the fluid flow in the secondary flow direction in the region near the plate. This tendency of the magnetic field may be due to Newtonian heating at the surface of the plate.

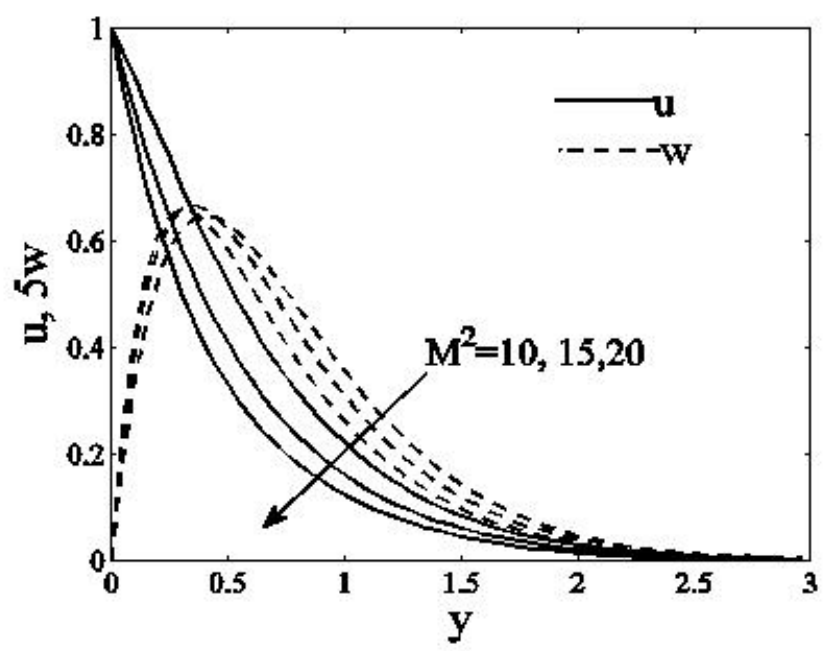

Fig.4. Velocity profiles when $\mathrm{G}_{\mathrm{r}}=5, m=0.5, K_{1}=0.4, \gamma=1, \mathrm{P}_{r}=0.71$ and $t=0.5$. 


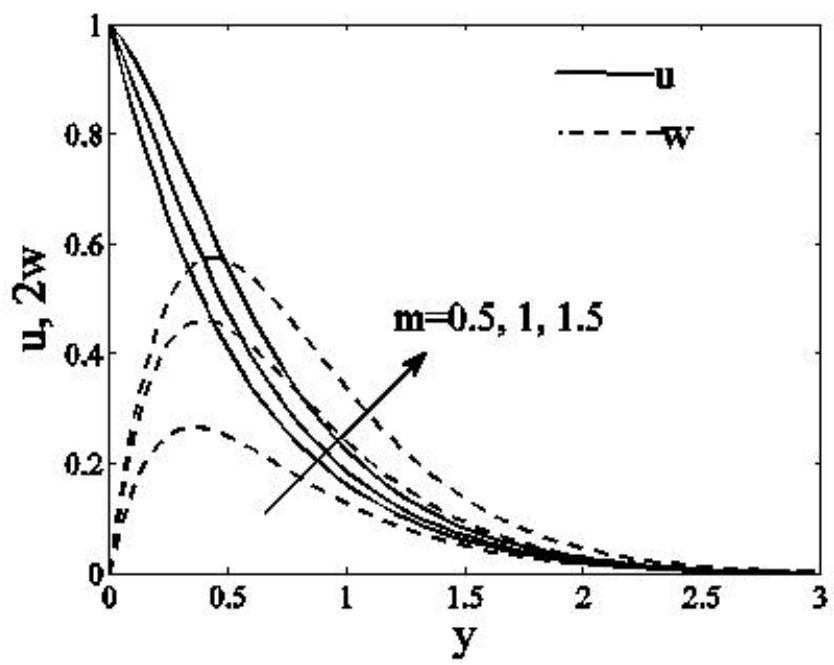

Fig.5. Velocity profiles when $\mathrm{G}_{\mathrm{r}}=5, M^{2}=15, K_{1}=0.4, \gamma=1, \mathrm{P}_{r}=0.71$ and $t=0.5$.

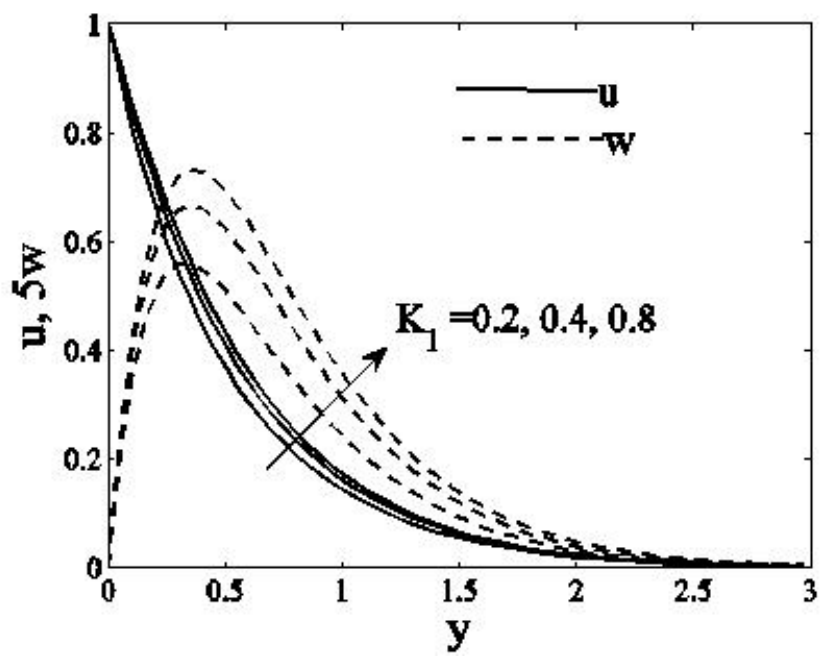

Fig.6. Velocity profiles when $\mathrm{G}_{\mathrm{r}}=5, M^{2}=15, m=0.5, \gamma=1, \mathrm{P}_{r}=0.71$ and $t=0.5$.

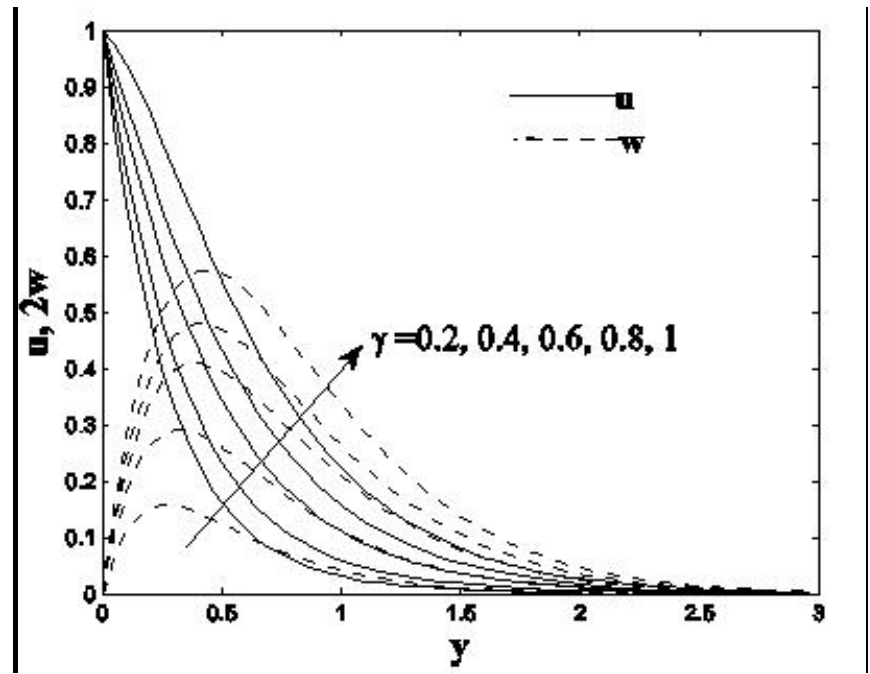

Fig.7. Velocity profiles when $\mathrm{G}_{\mathrm{r}}=5, M^{2}=15, m=0.5, K_{1}=0.4, \mathrm{P}_{r}=0.71$ and $t=0.5$. 
Figures 5 and 6 display the effects of Hall current and permeability of the medium on the primary and secondary fluid velocities. It is evident from Figures 5 and 6 that both $u$ and $w$ increase on increasing $m$ and $K_{l}$. This implies that Hall current tends to accelerate the fluid flow in both the primary and secondary flow directions throughout the boundary layer region. It may be noted that an increase $K_{l}$ implies that there is a decrease in the resistance of the porous medium. Due to this reason permeability of the medium tends to accelerate fluid velocities in both the primary and secondary flow directions throughout the boundary layer region. Figure 7 reveals the influence of Newtonian heating parameter on the primary and secondary fluid velocities. It is observed from Fig.7 that both $u$ and $w$ increase on increasing $\gamma$. This implies that Newtonian heating tends to accelerate fluid velocities in both the primary and secondary flow directions throughout the boundary layer region. It may be noted that as the Newtonian heating parameter increases, the density of the fluid decreases and the momentum boundary layer thickness increases and, as a result, the velocity increases within the boundary layer (Abid et al. [40]).

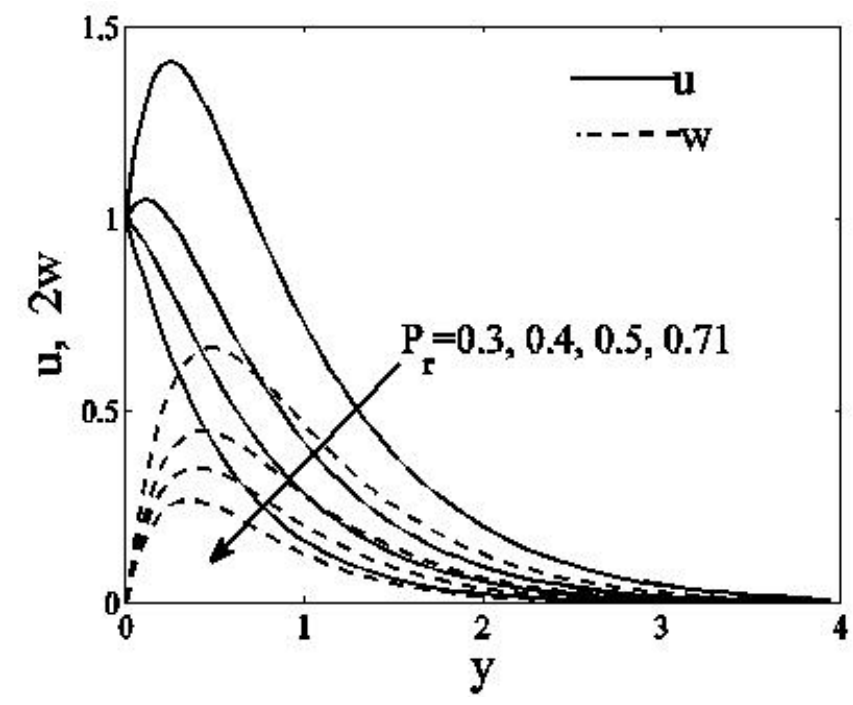

Fig.8. Velocity profiles when $\mathrm{G}_{\mathrm{r}}=5, M^{2}=15, m=0.5, K_{1}=0.4, \gamma=1$, and $t=0.5$.

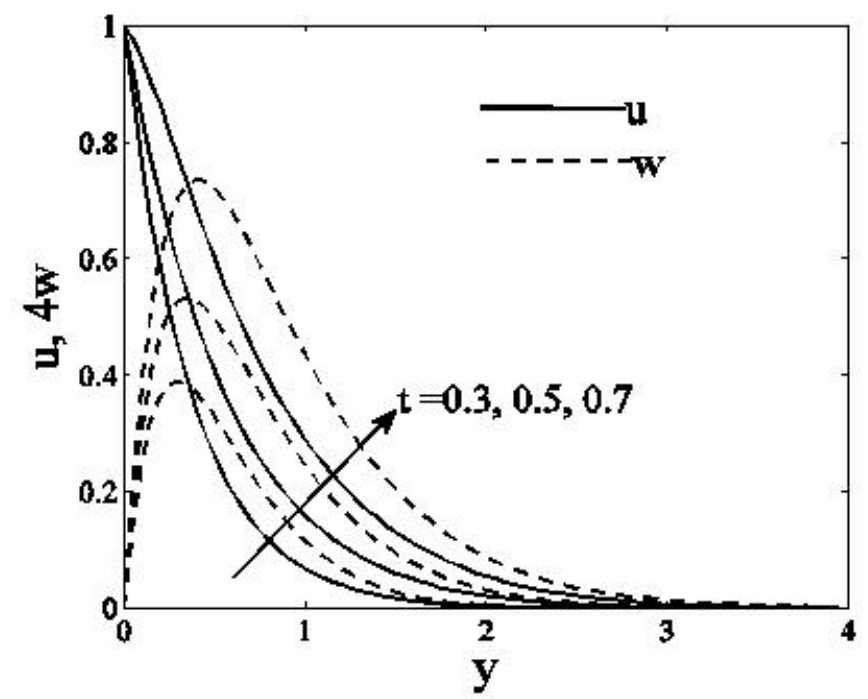

Fig.9. Velocity profiles when $\mathrm{G}_{\mathrm{r}}=5, M^{2}=15, m=0.5, K_{1}=0.4, \gamma=1$, and $\mathrm{P}_{r}=0.71$. 
Figures 8 and 9 depict the effects of thermal diffusivity and time on primary and secondary fluid velocities. It is noticed from Figures 8 and 9 that both the primary and secondary fluid velocities decrease on increasing the Prandtl number $\mathrm{P}_{r}$ whereas these fluid velocities increase on increasing time $t . \mathrm{P}_{r}$ is a measure of a relative strength of viscosity to the thermal diffusivity of the fluid, $\mathrm{P}_{r}$ decreases on increasing the thermal diffusivity. This implies that thermal diffusion tends to accelerate the fluid flow in both the primary and secondary flow directions throughout the boundary layer region. As time progresses, the fluid flow is getting accelerated in both the primary and secondary flow directions throughout the boundary layer region.

The numerical values of the primary skin friction $\tau_{x}$ and secondary skin friction $\tau_{z}$, are presented in a tabular form in Tabs 2 to 4 for various values of $m, M^{2}, \mathrm{G}_{r}, \mathrm{P}_{r}, \gamma$ and $t$. It is evident from Tab.2 that the secondary skin friction, i.e., $\tau_{z}$, increases on increasing either $M^{2}$ or $m$. The primary skin friction, i.e., $\tau_{x}$ increases on increasing $M^{2}$ whereas it decreases on increasing $m$ when $M^{2} \geq 15$. This implies that the magnetic field and Hall current tend to enhance the secondary skin friction. The magnetic field has a tendency to enhance the primary skin friction whereas Hall current has a tendency to reduce it when $M^{2} \geq 15$. It is worthy to note that there exists flow separation at the surface of the plate on increasing $m$ when $M^{2}=10$ and on increasing $M^{2}$ when $m=1.5$. It is noticed from Tab.3 that the secondary skin friction $\tau_{z}$, increases on increasing $G_{r}$ whereas it decreases on increasing $\mathrm{P}_{r}$. The primary skin friction $\tau_{x}$ decreases, attains a minimum and then increases in magnitude on increasing Gr when $\operatorname{Pr} \leq 0.5$ and it decreases on increasing $G_{r}$ when $\mathrm{P}_{r}=0.71 . \tau_{x}$ increases on increasing $\mathrm{P}_{r}$ when $\mathrm{Gr}=2$ and it decreases in magnitude, attains a minimum, and then increases on increasing $\mathrm{P}_{r}$ when $\mathrm{Gr}=5$ and 8 . This implies that the thermal buoyancy force and thermal diffusion tend to enhance the secondary skin friction. Thermal diffusion has a tendency to reduce the primary skin friction when $\mathrm{G}_{r}=2$ whereas the thermal buoyancy force has a tendency to reduce the primary skin friction when $\mathrm{P}_{r}=0.71$. It is also perceived from Tab.3 that there exists flow separation at the surface of the plate on increasing $G_{r}$ when $\mathrm{P}_{r} \leq 0.5$ and on increasing $\mathrm{P}_{r}$ when $\mathrm{G}_{r}=5$ and 8 . It is revealed from Tab.4 that $\tau_{x}$ decreases on increasing either $\gamma$ or $t$ whereas $\tau_{z}$, increases on increasing either $\gamma$ or $t$. This implies that Newtonian heating tends to reduce the primary skin friction whereas it has a reverse effect on the secondary skin friction. As time progresses, the primary skin friction is getting reduced whereas the secondary skin friction is getting enhanced.

Table 2. Skin friction when $\mathrm{G}_{r}=5, K_{l}=0.4, \mathrm{P}_{r}=0.71, \gamma=1$ and $t=0.5$.

\begin{tabular}{|l|c|c|c|c|c|c|}
\hline \multirow{2}{*}{$\begin{array}{l}m \downarrow \\
\rightarrow\end{array}$} & \multicolumn{3}{|c|}{$-\tau_{x}$} & \multicolumn{3}{|c|}{$\tau_{z}$} \\
\cline { 3 - 8 } & 10 & 15 & 20 & 10 & 15 & 20 \\
\hline 0.5 & 0.8573 & 1.6750 & 2.3578 & 0.8487 & 1.0376 & 1.1818 \\
\hline 1 & 0.2716 & 0.9809 & 1.5818 & 1.2529 & 1.5588 & 1.7892 \\
\hline 1.5 & -0.2318 & 0.3703 & 0.8950 & 1.3390 & 1.7081 & 1.9843 \\
\hline
\end{tabular}

Table 3. Skin friction when $m=0.5, M^{2}=15, K_{l}=0.4, \gamma=1$ and $t=0.5$.

\begin{tabular}{|l|c|c|c|c|c|c|c|c|}
\hline & \multicolumn{4}{|c|}{$-\tau_{x}$} & \multicolumn{4}{c|}{$\tau_{z}$} \\
\cline { 2 - 9 } $\mathrm{G}_{\mathrm{r} \downarrow} \quad \mathrm{P}_{\mathrm{r}} \rightarrow$ & 0.3 & 0.4 & 0.5 & 0.71 & 0.3 & 0.4 & 0.5 & 0.71 \\
\hline 2 & 0.6292 & 1.9136 & 2.4766 & 2.9977 & 1.1696 & 1.0130 & 0.9424 & 0.8758 \\
\hline 5 & -4.2461 & -1.0353 & 0.3723 & 1.6750 & 1.7723 & 1.3808 & 1.2043 & 1.0376 \\
\hline 8 & -9.1214 & -3.9841 & -1.7320 & 0.3523 & 2.3749 & 1.7486 & 1.4662 & 1.1995 \\
\hline
\end{tabular}


Table 4. Skin friction when $m=0.5, M^{2}=15, K_{l}=0.8, \mathrm{G}_{r}=5$ and $\mathrm{P}_{r}=0.71$.

\begin{tabular}{|l|c|c|c|c|c|c|c|c|}
\hline \multirow{2}{*}{$t \downarrow \quad \gamma \rightarrow$} & \multicolumn{4}{|c|}{$-\tau_{x}$} & \multicolumn{4}{c|}{$\tau_{z}$} \\
\cline { 2 - 9 } & 0.2 & 0.4 & 0.8 & 1 & 0.2 & 0.4 & 0.8 & 1 \\
\hline 0.3 & 3.5794 & 3.4016 & 2.8868 & 2.5081 & 0.8162 & 0.8367 & 0.8930 & 0.9323 \\
\hline 0.5 & 3.5128 & 3.2296 & 2.2813 & 1.4650 & 0.8306 & 0.8697 & 0.9930 & 1.0930 \\
\hline 0.7 & 3.4539 & 3.0669 & 1.5895 & 0.1238 & 0.8424 & 0.9003 & 1.1083 & 1.3020 \\
\hline
\end{tabular}

\section{Conclusions}

An unsteady hydromagnetic free convection flow through a uniform porous medium past an impulsively moving vertical plate with Newtonian heating and Hall effects is studied. It is found that Hall current, permeability of the medium, thermal buoyancy force, Newtonian heating and thermal diffusion tend to accelerate the fluid flow in both the primary and secondary flow directions throughout the boundary layer region. The magnetic field tends to retard the fluid flow in the primary flow direction throughout the boundary layer region whereas it tends to retard the fluid flow in the region away from the plate in the secondary flow direction. The fluid flow is getting accelerated in both the primary and secondary flow directions throughout the boundary layer region with the passage of time. The magnetic field and Hall current tend to increase the secondary skin friction. The magnetic field has a tendency to enhance the primary skin friction whereas Hall current has a tendency to reduce it when $M^{2} \geq 15$. The thermal buoyancy force and thermal diffusion tend to increase the secondary skin friction. Newtonian heating tends to reduce the primary skin friction whereas it has a reverse effect on the secondary skin friction.

\section{Nomenclature}

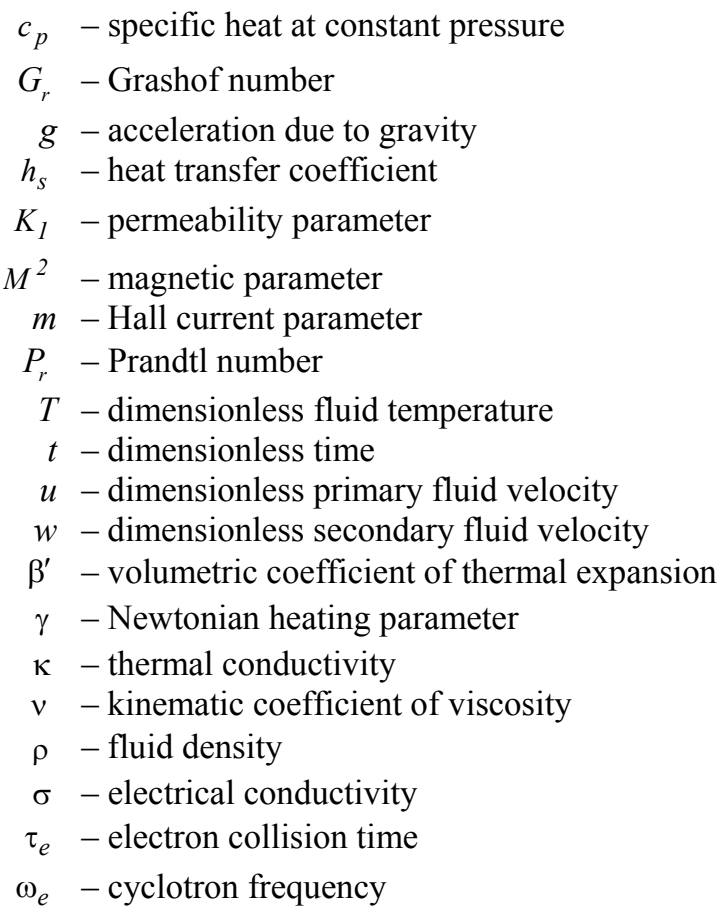




\section{References}

[1] Cheng P. and Minkowycz W.J. (1977): Free convection about a vertical flat plate embedded in a porous medium with application to heat transfer from a dike.- J. Geophys. Res., vol.82, pp.2040-2044.

[2] Nakayama A. and Koyama H. (1987): A general similarity transformation for combined free and forced convection flows within a fluid saturated porous medium. - ASME J. Heat Transf., vol.109, pp.1041-1045.

[3] Lai F.C. and Kulacki F.A. (1991): Non-Darcy mixed convection along a vertical wall in a saturated porous medium. - ASME J. Heat Transf., vol.113, pp.252-254.

[4] Bakier A.Y., Mansour M.A., Gorla R.S.R. and Ebiana A.B. (1997): Nonsimilar solutions for free convection from a vertical plate in porous media. - Heat Mass Transf., vol.33, pp.145-148.

[5] Nield D.A. and Bejan A. (2006): Convection in Porous Media, Third Ed., Springer. New York:

[6] Ingham D.B. and Pop I. (2005): Transport Phenomena in Porous Media. - vol. III, Elsevier, Oxford. U. K.

[7] Vafai K. (2005): Handbook of Porous Media. - Second Ed., Taylor \& Francis, New York.

[8] Pop I. and Ingham D.B. (2001): Convective Heat Transfer: Mathematical and Computational Modelling of Viscous Fluids and Porous Media., Pergamon. Oxford, U. K.

[9] Ingham D.B., Bejan A., Mamut E. and Pop I. (2004): Emerging Technologies and Techniques in Porous Media., Kluwer, Dordrecht, Netherlands.

[10] Bejan A., Dincer I., Lorente S., Miguel A.F. and Reis A.H. (2004): Porous and Complex Flow Structures in Modern Technologies, Springer, New York.

[11] Merkin J.H. (1994): Natural-convection boundary-layer flow on a vertical surface with Newtonian heating. - Int. J. Heat Fluid Flow, vol.15, pp.392-398.

[12] Lesnic D., Ingham D.B., and Pop I. (1999): Free convection boundary layer flow along a vertical surface in a porous medium with Newtonian heating.- Int. J. Heat Mass Transf., vol.42, pp.2621-2627.

[13] Lesnic D., Ingham D.B. and Pop I. (2000): Free convection from a horizontal surface in a porous medium with Newtonian heating. - J. Porous Med., vol.3, pp.227-235.

[14] Lesnic D., Ingham D.B., Pop I. and Storr C. (2004): Free convection boundary-layer flow above a nearly horizontal surface in a porous medium with Newtonian heating.- Heat Mass Transf., vol.40, pp.665-672.

[15] Salleh M.Z., Nazar R. and Pop I. (2009): Forced convection boundary layer flow at a forward stagnation point with Newtonian heating. - Chem. Eng. Comm., vol.196, pp.987-996.

[16] Salleh M.Z., Nazar R. and Pop I. (2010): Boundary layer flow and heat transfer over a stretching sheet with Newtonian heating. - J. Taiwan Inst. Chem. Engineers, vol.41, pp.651-655.

[17] Chaudhary R.C. and Jain P. (2006): Unsteady free convection boundary-layer flow past an impulsively started vertical plate with Newtonian heating. - Rom. J. Phys., vol.51, pp.911-925.

[18] Mebine P. and Adigio E.M. (2009): Unsteady free convection flow with thermal radiation past a vertical porous plate with Newtonian heating. - Turk. J. Phys., vol.33, pp.109-119.

[19] Narahari M. and Ishak A. (2011): Radiation effects on free convection flow near a moving vertical plate with Newtonian heating. - J. Appl. Sci., vol.11, pp.1096-1104.

[20] Narahari M. and Nayan M.Y. (2011): Free convection flow past an impulsively started infinite vertical plate with Newtonian heating in the presence of thermal radiation and mass diffusion.- Turk. J. Eng. Env. Sci., vol.35, pp.187-198.

[21] Olanrewaju A.M. and Makinde O.D. (2013): On boundary layer stagnation point flow of a nanofluid over a permeable flat surface with Newtonian heating.- Chem. Eng. Comm., vol.200, pp.836-852. 
[22] Steg L. and Sutton G.W. (1960): Prospects of MHD Power Generation. - Astronautics, Vol.5, pp.22-25.

[23] Womac G.J. (1969): MHD Power Generation. - London: Chapman and Hall.

[24] Shercliff J.A. (1962): The Theory of Electromagnetic Flow-Measurement. - Cambridge: CUP.

[25] Blake L.R. (1957): Conduction and induction pumps for liquid metals. - Proc. Inst. Elec. Engrs. London, vol.104A, pp.49-62.

[26] Marston C.H. (1966): MHD accelerator performance for specified interaction parameter. - AIAA Journal, vol.4, No.11, pp.2078-2079. doi: 10.2514/3.3858

[27] Christofilos N.C. (1958): Astron Thermonuclear Reactor. - Proc $2^{\text {nd }}$ UN Int. Conf. Peaceful Uses of Atomic Energy, Geneva, vol.32, pp.279-290.

[28] Raptis A.A. (1986): Flow through a porous medium in the presence of a magnetic field. - Int. J. Energy Res., vol.10, pp.97-100.

[29] Jha B.K. (1991): MHD free convection and mass-transform flow through a porous medium. - Astrophys. Space Sci., vol.175, pp.283-289.

[30] Chamkha A.J. (1997): Transient MHD free convection from a porous medium supported by a surface. Fluid/Particle Separation Journal, vol.10, pp.101-107.

[31] Kim Y.J. (2000): Unsteady MHD convective heat transfer past a semi-infinite vertical porous moving plate with variable suction. - Int. J. Eng. Sci., vol.38, pp.833-845.

[32] Ibrahim F.S., Hassanien I.A. and Bakr A.A. (2004): Unsteady magnetohydrodynamic micro-polar fluid flow and heat transfer over a vertical porous plate through a porous medium in the presence of thermal and mass diffusion with a constant heat source. - Canad. J. Phys., vol.82, pp.775-790.

[33] Chamkha A.J. (2004): Unsteady MHD convective heat and mass transfer past a semi-infinite vertical permeable moving plate with heat absorption. - Int. J. Eng. Sci., vol.42, pp.217-230.

[34] Seth G.S., Ansari Md.S. and Nandkeolyar R. (2011): MHD natural convection flow with radiative heat transfer past an impulsively moving plate with ramped wall temperature. - Heat Mass Transf., vol.47, pp.551-561.

[35] Mahmoud M.A.A. (2009): Thermal radiation effect on unsteady MHD free convection flow past a vertical plate with temperature dependent viscosity. - Canad. J. Chem. Eng., vol.87, pp.47-52.

[36] Ogulu A. and Makinde O.D. (2008): Unsteady hydromagnetic free convection flow of a dissipative and radiating fluid past a vertical plate with constant heat flux. - Chem. Eng. Comm., vol.196, pp.454-462.

[37] Chamkha A.J., Mohamed R.A. and Ahmed S.E. (2011): Unsteady MHD natural convection from a heated vertical porous plate in a micropolar fluid with Joule heating, chemical reaction and radiation effects. - Meccanica, vol.46, pp.399-411.

[38] Mohamed R.A., Osman A.N.A. and Abo-Dahab S.M. (2013): Unsteady MHD double-diffusive convection boundary-layer flow past a radiate hot vertical surface in porous media in the presence of chemical reaction and heat sink. - Meccanica, vol.48, pp.931-942.

[39] Singh G. and Makinde O.D. (2012): Computational dynamics of MHD free convection flow along an inclined plate with Newtonian heating in the presence of volumetric heat generation. - Chem. Eng. Comm., vol.199, pp.11441154.

[40] Abid H., Ismail Z., Khan I., Hussein A.G. and Shafie S. (2014): Unsteady boundary layer MHD free convection flow in a porous medium with constant mass diffusion and Newtonian heating. - Eur. Phys. J. Plus, vol.129, p.46.

[41] Sherman A. and Sutton G.W. (2006): Engineering Magnetohydrodynamics. - New York: Dover Pub Inc.

[42] Fife J. (1998): Hybrid-PIC Modeling and electrostatic probe survey of Hall thrusters. - PhD Thesis, Department of Aeronautics and Astronautics, MIT, USA.

[43] Shang J.S., Surzhikov S.T., Kimmel R., Gaitonde D., Menart J. and Hayes J. (2005): Mechanisms of plasma actuators for hypersonic flow control. - Prog. Aerosp. Sci., vol.41, pp 642-668. 
[44] Kholshchevnikova E.K. (1966): Influence of the Hall effect on the characteristics of a MHD generator with two pairs of electrodes. - J. Appl. Mech. Tech. Phys., vol.7, No.4, pp.48-54.

[45] Michaeli K., Tikhonov K. S. and Finkel'stein A.M. (2012): Hall effect in superconducting films. - Physical Review, B 86014515.

[46] Davidson P.A. (1999): Magnetohydrodynamics in materials processing.- Annual Review Fluid Mech. vol.31, pp.273-300.

[47] Hardianto T., Sakamoto N. and Harada N. (2008): Computational study of diagonal channel magnetohydrodynamic power generation. - Int. J. Energy Technol. Policy, vol.6, pp.96-111.

[48] Mathon Ph., Nouri A., Alemany A., Chopart J.P., Sobolik V. and Baaziz D. (2009): Electro-chemical processes controlled by high magnetic fields: application to MHD sea water propulsion.- Magnetohydrodynamics, vol.45, pp.281-288.

[49] Van Wie D.M. (2005): Future Technologies - Application of Plasma Devices for Vehicle Systems. - The Johns Hopkins University, Applied Physics Laboratory - Laurel, Maryland, USA - NATO Document.

[50] Morley N.B., Malang S. and Kirillov I. (2005): Thermofluid Magnetohydrodynamic issues for liquid breeders.Fusion Sci. Tech., vol.47, pp.488-501.

[51] Pop I. and Watanabe T. (1994): Hall effect on magnetohydrodynamic free convection about a semi-infinite vertical flat plate. - Int. J. Eng. Sci., vol.32, pp.1903-1911.

[52] Abo-Eldahab E.M. and Elbarbary E.M.E. (2001): Hall current effect on magnetohydrodynamic free-convection flow past a semi-infinite vertical plate with mass transfer. - Int. J. Eng. Sci. vol.39, pp.1641-1652.

[53] Takhar H.S., Roy S. and Nath G. (2003): Unsteady free convection flow over an infinite vertical porous plate due to the combined effects of thermal and mass diffusion, magnetic field and Hall currents. - Heat Mass Transf., vol.39, pp.825-834.

[54] Saha L.K., Siddiqa S. and Hossain M.A. (2011): Effect of Hall current on MHD natural convection flow from vertical permeable flat plate with uniform surface heat flux. - Appl. Math. Mech., vol.32, pp.1127-1146.

[55] Seth G.S., Mahato G.K. and Sarkar S. (2013): Effects of Hall current and rotation on MHD natural convection flow past an impulsively moving vertical plate with ramped temperature in the presence of thermal diffusion with heat absorption. - Int. J. Energy Tech., vol.5, No.16, pp.1-12.

[56] Cramer K.R. and Pai S.I. (1973): Magnetofluiddynamics for Engineers and Applied Physicists. - New York: McGraw Hill Book Company.

[57] Abid H., Khan I. and Shafie S. (2013): An exact analysis of heat and mass transfer past a vertical plate with Newtonian heating. - J Appl. Math., vol.2013, Article ID 434571, pp.1-9.

[58] de Hoog F.R., Knight J.H. and Stokes A.N. (1982): An improved method for numerical inversion of Laplace transforms. - S.I.A.M. J. Sci. and Stat. Comput., vol.3, pp.357-366.

[59] Hollenbeck K.J. (1998): Invlap. M: A matlab function for numerical inversion of Laplace transforms by the de Hoog algorithm. - http://www.isva.dtu.dk/staff/karl/invlap.htm.

[60] Carnahan B., Luther H.A. and Wilkes J.O. (1969): Applied Numerical Methods. John Wiley and Sons, New York

[61] Antia H.M. (1991): Numerical Methods for Scientists and Engineers. - Tata McGraw-Hill Publishing Co Ltd, New Delhi, India. 